\title{
On-Wafer Series-Through Broadband Measurement of Sub-fF55nm MOS RF Voltage-Tunable Capacitors
}

\author{
K. Daffé, G. Dambrine, Fellow, IEEE, C. Durand, C. Gaquière and K. Haddadi, Member, IEEE
}

\begin{abstract}
The objective of this letter oriented towards microwave measurement of high impedance devices using a conventional on-wafer probe station is multiple. First, we provided a quantification of the measurement uncertainty inherent to the set-up when measuring capacitors in the range 0.01-10 fF using both reflection and transmission methods up to 50 GHz. In particular, we demonstrate a clear improvement when using transmission method in series-through configuration. As a practical demonstration, on-wafer MOS voltage-tunable capacitors with capacitances ranging from 0.85 to $1.15 \mathrm{fF}$ are extracted with uncertainties of 130 and 2 aF respectively for both reflection and transmission measurements at $10 \mathrm{GHz}$. Capacitance fluctuation related to the technological process in the order of $20 \mathrm{aF}$ is then exemplary demonstrated using the series-through configuration.
\end{abstract}

Index Terms-sub-fF MOS varactor, measurement accuracy, on-wafer calibration, vector network analyzer (VNA), two-port measurement, coplanar waveguide (CPW).

\section{INTRODUCTION}

$\mathrm{T}^{0}$ $O$ foster the progress of miniaturization of electronic circuits, new metrological issues related to the electrical characterization of nanoelectronic devices must be addressed[1].The microwave characterization of extremely high impedance devices such as Attofarad $(\mathrm{aF})$ capacitors[2] and 1D based devices [3] is still challenging. In particular, the microwave calibrated measurement of RF voltage-tunable integrated capacitors, i.e. varactors with capacitance values ranging from few $\mathrm{fF}$ to sub-fF, is required to address RF tuning optimization within multi-bands wireless devices applications[4]-[5]. Indeed, a slight discrepancy of the capacitance value can impact fatally the operating frequency stability of the targeted applications. Whereas available design kits are built up with HICUM models that consider process variability through material and topographic characterizations, there is an urgent need to assess the traceability and the variability of these models through actual electrical measurements. The critical issue is related to the measurement

Manuscript received December 28, 2017; revised May 3, 2018. This work is performed under Nano2017 (http://www.st.com/web/en/press/c2727) and EMPIR Planarcal projects (http://www.planarcal.ptb.de). This work used the facilities within the EQPX ExCELSiOR(www.excelsior-ncc.eu).funded by the National Research Agency (ANR).

K. Daffé, K. Haddadi, C. Gaquière and G. Dambrine are with Univ. Lille1, CNRS, UMR 8520 - IEMN, F-59000 Lille, France. (e-mail : kamel.haddadi@univ-lille.fr).

C. Durand is with STMicroelectronics, F-38926 Crolles, France. uncertainty, which degrades with device downscaling and the raise of operating frequency. Near-field microwave microscopy and nano-robotic micro-probing station [6]are interesting approaches but they are not suitable for industrial test in the general context of the silicon industry.

In this letter, a conventional device characterization set-up, i.e. a vector network analyzer (VNA) connected to a pair of GSG probes, is proposed in a CPW series-through measurement configuration to tackle the issue of extreme impedance microwave measurement. The Section II provides the theoretical background for capacitance uncertainty estimation using the proposed series-through configuration. In Section III, MOS RF voltage-tunable capacitors with capacitances ranging from 0.85 to $1.15 \mathrm{fF}$ are determined through reflection and transmission coefficient measurements. In particular, measurement errors inherent to the microwave instrumentation, the probe repeatability and the manufacturing process are quantified.

\section{Capacitance UnCERTAinty}

The measurement uncertainty estimation starts from the commonly applied two-port error models corresponding to the series-through configurations in which an ideal capacitance $C$ is considered as the device under test (DUT) (Fig. 1.).

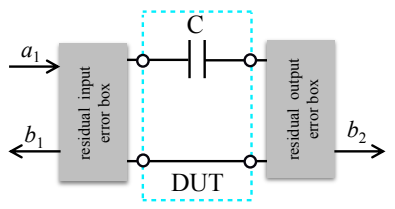

(a)

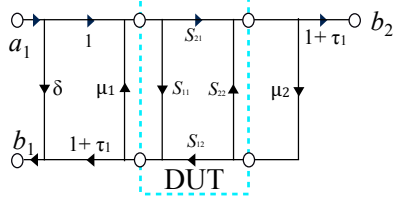

(b)
Fig. 1.VNA two-port series-through capacitance measurement configuration (a) and associated flow graph (b).

The quantities $a_{i}$ and $b_{i}(\mathrm{i}=1,2)$ denotes the incoming and emerging traveling waves at the measurement planes respectively. The terms $\delta, \tau_{1}, \mu_{1}, \tau_{2}$ and $\mu_{2}$ are the postcalibration residual calibration error terms, namely postcalibration residual directivity, reflection tracking, source match, transmission tracking and load match of the calibrated VNA that relate the difference between the scattering parameters obtained for two different calibrations. The crosstalk leakage term is neglected because of its very small contribution less than $10^{-5}$. The complex values of the postcalibration residual error terms are extracted as the standard deviations in respect to the mean values related to 7 successive vector calibrations, i.e. line-reflect-reflect-match (LRRM). These non-systematic residual errors that include the random (noise, connector repeatability, probe contact 
repeatability), the drift and stability effects between measurements are quantified as follow.

STEP 1:An N5225A PNA VNA from ${ }^{\circledR}$ Keysight is used with frequency step set to $100 \mathrm{MHz}$ (491 points) in the frequency range spanning from $50 \mathrm{MHz}$ to $50 \mathrm{GHz}$. The RF signal power is set to $0 \mathrm{dBm}$ and an intermediate frequency bandwidth (IFBW) of $100 \mathrm{~Hz}$ is used. The positioner is equipped with a $100 \mu \mathrm{m}$ pitch GSG ${ }^{\circledR}$ Infinity probe. A microwave cable $(2.4 \mathrm{~mm})$ from ${ }^{\circledR}$ Gore with high phase and amplitude stability with flexure is used to connect the probe to the VNA. The Impedance Standard Substrate (ISS) reference is \#101-190 from ${ }^{\circledR}$ Cascade Microtech. The mechanical onwafer station is a MPS150 from ${ }^{\circledR}$ Cascade. The experimental post-calibration residual error terms are shown in Fig. 2.
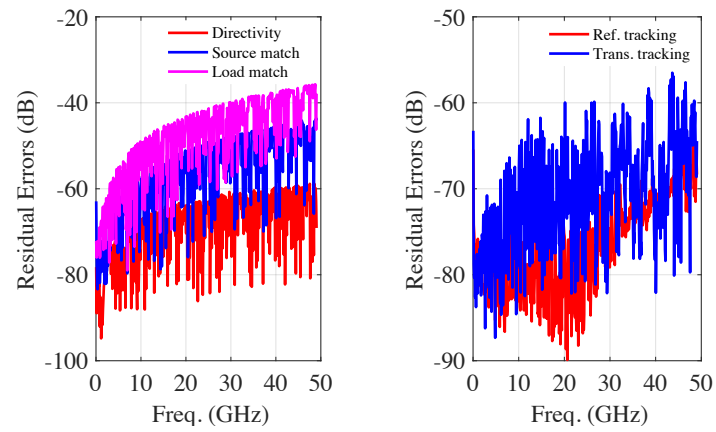

Fig. 2.Standard deviations $(2 \sigma)$ of measurement error terms versus frequency.

STEP 2: Flow graphs from Fig. 1 using post-calibration residual error terms are solved to determine the total system magnitude $\left(\left|\Delta \mathrm{S}_{11}\right|\right.$ and $\left.\left|\Delta \mathrm{S}_{21}\right|\right)$ and phase $\left(\Delta \Phi_{11}\right.$ and $\left.\Delta \Phi_{21}\right)$ uncertainties by the following forms [7].

$$
\begin{gathered}
\left|\Delta \mathrm{S}_{11}\right| \approx \delta+\tau_{1}\left|\mathrm{~S}_{11}\right|+\mu_{1}\left|\mathrm{~S}_{11}\right|^{2} \\
\Delta \Phi_{11} \approx \arcsin \left(\frac{\left|\Delta \mathrm{S}_{11}\right|}{\left|\mathrm{S}_{11}\right|}\right) \\
\left|\Delta S_{21}\right| \approx\left(\tau_{2}+\mu_{1}\left|S_{11}\right|+\mu_{2}\left|S_{22}\right|+\mu_{1} \mu_{2}\left|S_{12}\right|\left|S_{21}\right|\right)\left|S_{21}\right| \\
\Delta \Phi_{21} \approx \arcsin \left(\frac{\left|\Delta \mathrm{S}_{21}\right|}{\left|\mathrm{S}_{21}\right|}\right)
\end{gathered}
$$

STEP 3: The admittance terms of the Y-matrix of the DUT can be derived from $S$-parameters by

$$
\begin{gathered}
\mathrm{Y}_{11}=\frac{1}{2 Z_{0}} \frac{1-\mathrm{S}_{11}}{\mathrm{~S}_{11}}=\frac{1}{2 Z_{0}} \frac{\cos \phi_{11}-\left|\mathrm{S}_{11}\right|-j \sin \phi_{11}}{\left|\mathrm{~S}_{11}\right|} \\
\mathrm{Y}_{21}=\frac{1}{2 Z_{0}} \frac{\mathrm{S}_{21}}{1-\mathrm{S}_{21}} \\
=\frac{1}{2 Z_{0}} \frac{\left|\mathrm{S}_{21}\right| \cos \phi_{21}-1+\mathrm{j}\left|\mathrm{S}_{21}\right| \sin \phi_{21}}{1-2\left|\mathrm{~S}_{21}\right| \cos \phi_{21}+\left|\mathrm{S}_{21}\right|^{2}}
\end{gathered}
$$

STEP 4: Effective capacitance $C_{i j}$ and corresponding propagated uncertainty $\Delta C_{i j}$ are derived by

$$
\mathrm{C}_{11}=\frac{\left|\operatorname{Im}\left(\mathrm{Y}_{11}\right)\right|}{\omega}=\frac{1}{2 Z_{0}} \frac{\sin \phi_{11}}{\left|\mathrm{~S}_{11}\right|}
$$

$$
\begin{gathered}
\mathrm{C}_{21}=\frac{\left|\mathrm{Im}\left(\mathrm{Y}_{21}\right)\right|}{\omega}=\frac{1}{2 Z_{0}} \frac{\left|\mathrm{S}_{21}\right| \sin \phi_{21}}{1-2\left|\mathrm{~S}_{21}\right| \cos \phi_{21}+\left|\mathrm{S}_{21}\right|^{2}} \\
\Delta \mathrm{C}_{\mathrm{ij}}=\sqrt{\left(\frac{\partial \mathrm{C}_{\mathrm{ij}}}{\partial\left|\mathrm{S}_{\mathrm{ij}}\right|}\left|\Delta \mathrm{S}_{11}\right|\right)^{2}+\left(\frac{\partial \mathrm{C}_{\mathrm{ij}}}{\partial \phi_{\mathrm{ij}}} \phi_{\mathrm{ij}}\right)^{2}}
\end{gathered}
$$

where $\omega$ denotes the angular frequency.

The resulting capacitance uncertainties from both case studies are plotted in Fig. 3 at 1, 10 and $50 \mathrm{GHz}$.

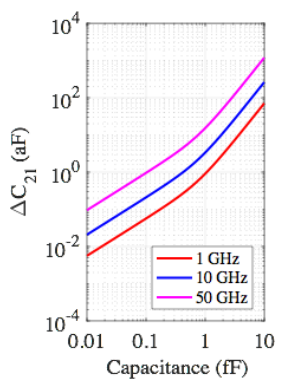

(a)

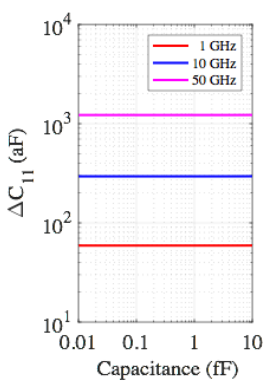

(b)

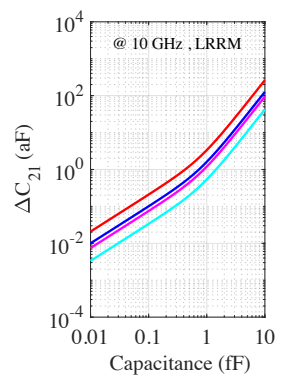

(c)
Fig.3. (a) and (b) Calculated capacitance uncertainties as a function of the capacitance. (c) Impact of the individual residual error terms on the capacitance uncertainty $-\mathrm{f}=10 \mathrm{GHz}(-$ full ; - Source match ; - Load match ; - Transmission tracking).

Fig. 3 highlights the performance of the two-port seriesthrough configuration in terms of capacitance uncertainty reduction. In the capacitances range considered, the uncertainty is reduced by a factor of nearly 20 when the capacitance is extracted from $\mathrm{S}_{21}$ in regards with the reflection coefficient measurement method. For example, the capacitance uncertainty value for a $1 \mathrm{fF}$ calculated at $10 \mathrm{GHz}$ is $5 \mathrm{aF}$ for $\Delta C_{21}$ while it reaches the value of $101 \mathrm{aF}$ for $\Delta C_{11}$. It has to be mentioned that the degradation of the capacitance uncertainty is mainly caused by the residual directivity error term when considering $\Delta C_{11}$. In other words, the two-port series-through configuration benefits from the excellent dynamic range performance of the VNA as the configuration is not offset by the directivity.

\section{EXPERIMENTAL VALIDATION}

As a demonstration, sub-fF MOS RF voltage-tunable capacitors from STMicroelectronics ${ }^{\mathrm{TM}}$ were considered (Fig. 4). The devices were biased from -2.5 to $2.5 \mathrm{~V}$ to achieve capacitance values ranging from $0.85 \mathrm{fF}$ to $1.15 \mathrm{fF}$. The measured $S$-parameters were mathematically corrected using a LRRM calibration algorithm [Fig. 5(a)]. An open/short deembedding procedure is also used to remove RF pad (YPAD), the access line $\left(\mathrm{Z}_{\mathrm{LINE}}\right)$ and the metal vias $\left(\mathrm{Z}_{\mathrm{VIA}}\right)$ contributions [Fig. 4(c)] [10]. $S_{11}$ data show high impedance mismatch $\left(\left|S_{11}\right|\right)$, i.e. the effective capacitance $\mathrm{C}_{11}$ is mainly related to the phase-shift [equation (7)]. $S_{21}$ data together with relation (8) show that measured transmission coefficient $\left|S_{21}\right|$ vary with the voltage whereas the phase-shift $\Phi_{21}$ remains nearly constant. At the first order, by neglecting the denominator term of (8), the effective capacitance can be determined 
directly by $C_{21} \approx \operatorname{Im}\left(S_{21}\right) / 2 Z_{0} \omega$. Indeed, Fig. 5(b) shows the effective capacitances determined using (7) and (8) together with the approximate capacitance discussed above for $\mathrm{V}=2.5 \mathrm{~V} . \mathrm{C}_{11}$ clearly shows a non-constant frequency behavior. In contrast, $\mathrm{C}_{21}$ indicates a flat response up to 25 GHz. Above $25 \mathrm{GHz}$, the model using a single capacitance C is no more available (slope of the curve) and the VNA becomes more sensitive to stochastic measurement errors (fluctuations of the signal [8]).

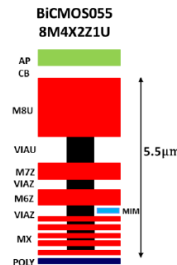

(a)

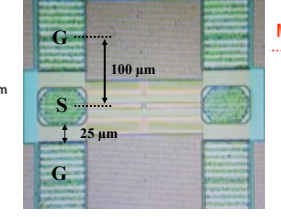

(b)

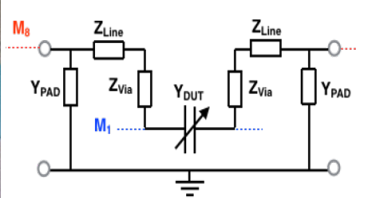

(c)
Fig.4. STMicroelectronics BEOL of the $55 \mathrm{~nm}$ SiGe BiCMOS Technology. (b) Micrograph of the varactor. (c) Equivalent electrical circuit model.
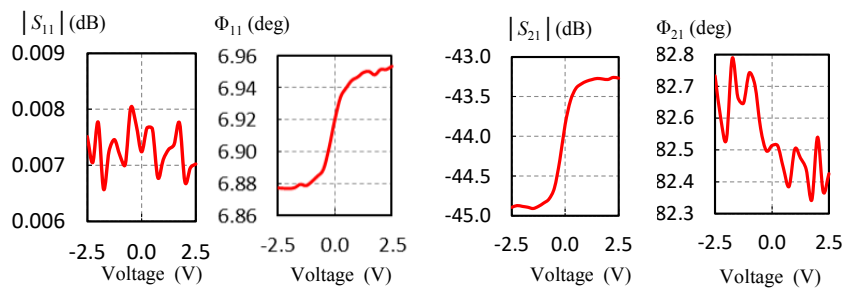

(a)

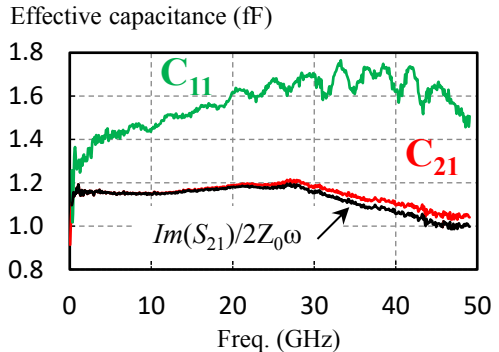

(b)

Fig. 5. (a) Measured $S$-parameters versus varactor voltage $-\mathrm{f}=10 \mathrm{GHz}$. (b) Effective capacitance versus frequency for $\mathrm{V}=2.5 \mathrm{~V}$.

The measurement uncertainty imputable to the probe contact repeatability $\left(\Delta \mathrm{C}_{11}, \Delta \mathrm{C}_{21}\right)$ was highlighted by performing six capacitance-voltage measurements of the same device. The time duration for the six measurements was less than $15 \mathrm{mn}$ [Fig. 6(a)]. For a bias voltage of $2.5 \mathrm{~V}$, the corresponding values are $\Delta \mathrm{C}_{11}=130 \mathrm{aF}$ and $\Delta \mathrm{C}_{21}=2 \mathrm{aF}$. Finally, measurement uncertainty relating to the process variability is investigated through die-to-die measurements on six different wafers location [Fig. 6(b)]. The corresponding errors $\left(\Delta \mathrm{C}_{11}, \Delta \mathrm{C}_{21}\right)$ for a biased voltage of $2.5 \mathrm{~V}$ are respectively 430 and $20 \mathrm{aF}$. From these two experiments, considering $\mathrm{C}_{21}$, the capacitance fluctuation due to the process variability $(20 \mathrm{aF})$ is 10 times bigger than the capacitance uncertainty $(2 \mathrm{aF})$. Therefore, the measurement can be used to assess the capacitance fluctuation accurately related to the process variability. On the other hand, considering $\mathrm{C}_{11}$, the capacitance uncertainty of $130 \mathrm{aF}$ is bigger that the capacitance fluctuation due to the process variability. Therefore, process variability cannot be studied by the reflection method.

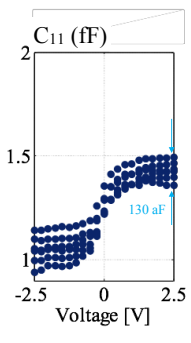

(a)

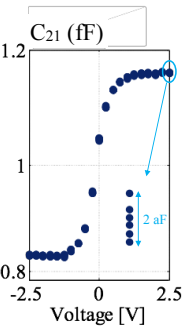

a)

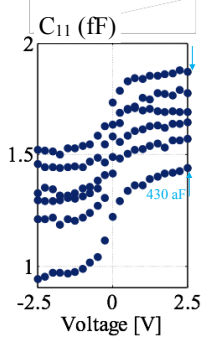

(b)

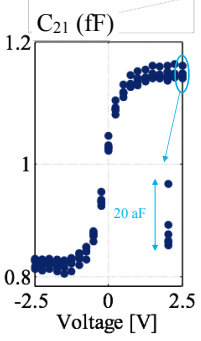

Fig. 6. (a) Measured effective capacitance for (a) six successive measurements and (b) for six successive locations $-\mathrm{f}=10 \mathrm{GHz}$.

\section{CONCLUSION}

We have estimated theoretically the measurement accuracy of fF capacitance extracted from reflection and transmission coefficient measurement considering an ideal capacitance connected in series-through configuration. The capacitance measurement error less than $1 \%$ is experimentally confirmed in the case of a $1 \mathrm{fF}$ varactor embedded in two-port seriesthrough configuration dealing with the transmission coefficient measurement. In contrast, the measurement error is about $8 \%$ when the capacitance is extracted from reflection coefficient measurement.

\section{REFERENCES}

[1] "The International Technology Roadmap for Semiconductors", Executive summary, 2013.

[2] R. Debroucke et al, "Sub-femtoFarad MOS varactor characterization tools," 40th European Microwave Conference, Paris, 2010, pp. 783786.

[3] H. Happy, K. Haddadi, D. Theron, T. Lasri, et G. Dambrine, "Measurement techniques for RF nanoelectronic devices: new equipment to overcome the problems of impedance and scale mismatch", IEEE Microw. Mag., vol. 15, n 1, pp. 30-39, Jan. 2014.

[4] J. He et al, "A 20-GHz VCO for PLL synthesizer in $0.13-\mu \mathrm{m}$ BiCMOS", in 2012 IEEE Intern. Symp. Radio-Frequency Integration Tech. (RFIT), 2012, pp. 231-233.

[5] C. Lee et al, "A 1.2V 37-38.5 GHz 8-phase clock generator in $0.13 \mathrm{um}$ CMOS technology", in 2006 Symposium on VLSI Circuits, Digest of Technical Papers, 2006, pp. 27-28.

[6] K. Haddadi et al, "Robotic on-wafer probe station for microwave characterization in a scanning electron microscope", in 2015 IEEE MTT-S International Microwave Symposium, 2015, pp. 1-3.

[7] D. K. Rytting, "Network analyzer accuracy overview ", in $58^{\text {th }}$ ARFTG Conference Digest, 2001, vol. 40, pp. 1-13.

[8] K. Daffé, G. Dambrine, F. von Kleist-Retzow, et K. Haddadi, "RF wafer probing with improved contact repeatability using nanometer positioning", in 2016 87th ARFTG Microwave Measurement Conference (ARFTG), 2016, pp. 1-4.

[9] P. Chevalier et al, "A $55 \mathrm{~nm}$ triple gate oxide 9 metal layers SiGe BiCMOS technology featuring $320 \mathrm{GHz} \mathrm{f} \mathrm{T} / 370 \mathrm{GHz}$ f MAX HBT and high-Q millimeter-wave passives," $60^{\text {th }}$ IEEE International Electron Devices Meeting (IEDM), San Francisco, CA, Dec. 2014, pp. 3-9.

[10] L. Carbonero, R. Joly, G. Morin and B. Cabon, "On-wafer highfrequency measurement improvements," IEEE International Conf. on Microelectronic Test Structures, San Diego, CA, 1994, pp. 168-173. 